\title{
Paraganglioma Cardíaco. Reporte de un caso
}

\author{
Enrique Seguel ${ }^{1,2}$, Roberto González ${ }^{1,2}$, Aleck Stockins ${ }^{1,2}$, Oriana Chavez ${ }^{3}$, Claudia Cabezas ${ }^{4,5}$. \\ 1. Hospital Guillermo Grant Benavente de Concepción, Centro Cardiovascular. \\ 2. Universidad de Concepción, Faculta de Medicina, Departamento de Cirugía, \\ 3. Universidad de Concepción, Alumna de Medicina. \\ 4. Hospital Guillermo Grant Benavente de Concepción, Servicio Anatomía Patológica. \\ 5. Universidad Católica de la Santísima Concepción, Facultad de Medicina
}

\section{Cardiac Paraganglioma: case report}

A 49-year-old woman presented with dyspnea and palpitations, leading to Functional Class III.An echocardiogram showed a heterogeneous mass adhered to the right heart cavities. This was confirmed by NMR. A large right coronary artery was occluded in relation to the tumor, which was hyper vascularized. Resection of the tumor was performed; the right ventricular wall was sutured, and an atrial defect was closed using pericardial tissue. Post operative course was uneventful and she was asymptomatic 4 years after surgery.

Keywords: Cardiac tumors, cardiac paraganglioma, paraganglioma. 


\section{Introducción:}

Los tumores cardiacos son infrecuentes. La incidencia de los tumores primarios del corazón (TCP) reportada en necropsias sólo llega hasta un $0.05 \%$. Los tumores metastásicos, en cambio, son 20 veces más comunes $\left(1 \%\right.$ en necropsias $\left.{ }^{1,2}\right)$

Entre el 70 y el $90 \%$ de los TCP en adultos son benignos, siendo el más común el mixoma atrial (50\%), seguido del lipoma (20\%), del fibroelastoma papilar $(15 \%)$ y del angioma (5\%). En el caso de los niños, el tumor más frecuente es el rabdomioma. ${ }^{3-5}$

Cuando presentan síntomas, el más común es la disnea, pero también pueden cursar con embolias, arritmias, dolor torácico, síntomas constitucionales e incluso muerte súbita. ${ }^{3,4}$

El paraganglioma cardíaco es extremadamente raro, con una frecuencia sólo de 0,001 a $0.003 \%$. Se presenta con mayor frecuencia en mujeres, entre la cuarta y quinta década de la vida, tiene un comportamiento incierto, pudiendo ser maligno en un $10 \%$ de los $\operatorname{casos}^{6-9}$.

\section{Caso Clínico:}

Mujer de 49 años procedente de Curicó, con antecedentes de hipertensión arterial en tratamiento con enalapril, tabaquismo activo y obesidad (IMC 32). Consulta por disnea y palpitaciones de dos años de evolución, progresiva hasta llegar a capacidad funcional III.

Un ecocardiograma de superficie mostró una masa heterogénea, encapsulada, de $6 \times 5 \mathrm{~cm}$, adosada a pared libre del ventrículo y aurícula derechos. La función sistólica del ventrículo izquierdo era normal, con una fracción de 55\%. Una tomografía computada de tórax y abdomen confirmó la presencia de una masa en surco aurículo - ventricular derecho, aparentemente extra cardíaca.

La resonancia nuclear magnética mostró una masa sólida en surco aurículo - ventricular derecho de 8 × 7 × 2 $\mathrm{cm}$, asociada a derrame pericárdico (Figura 1).

No se observaron otros tumores en los exámenes de imágenes.

Se completó su estudio con una angiografía coronaria, que mostró una arteria coronaria derecha es de gran de-

FIGURA1: Resonancia Nuclear magnética cardiaca en la que se observa una masa en el surco aurículo - ventricular derecho, la que comprime tanto a la aurícula como al ventrículo.

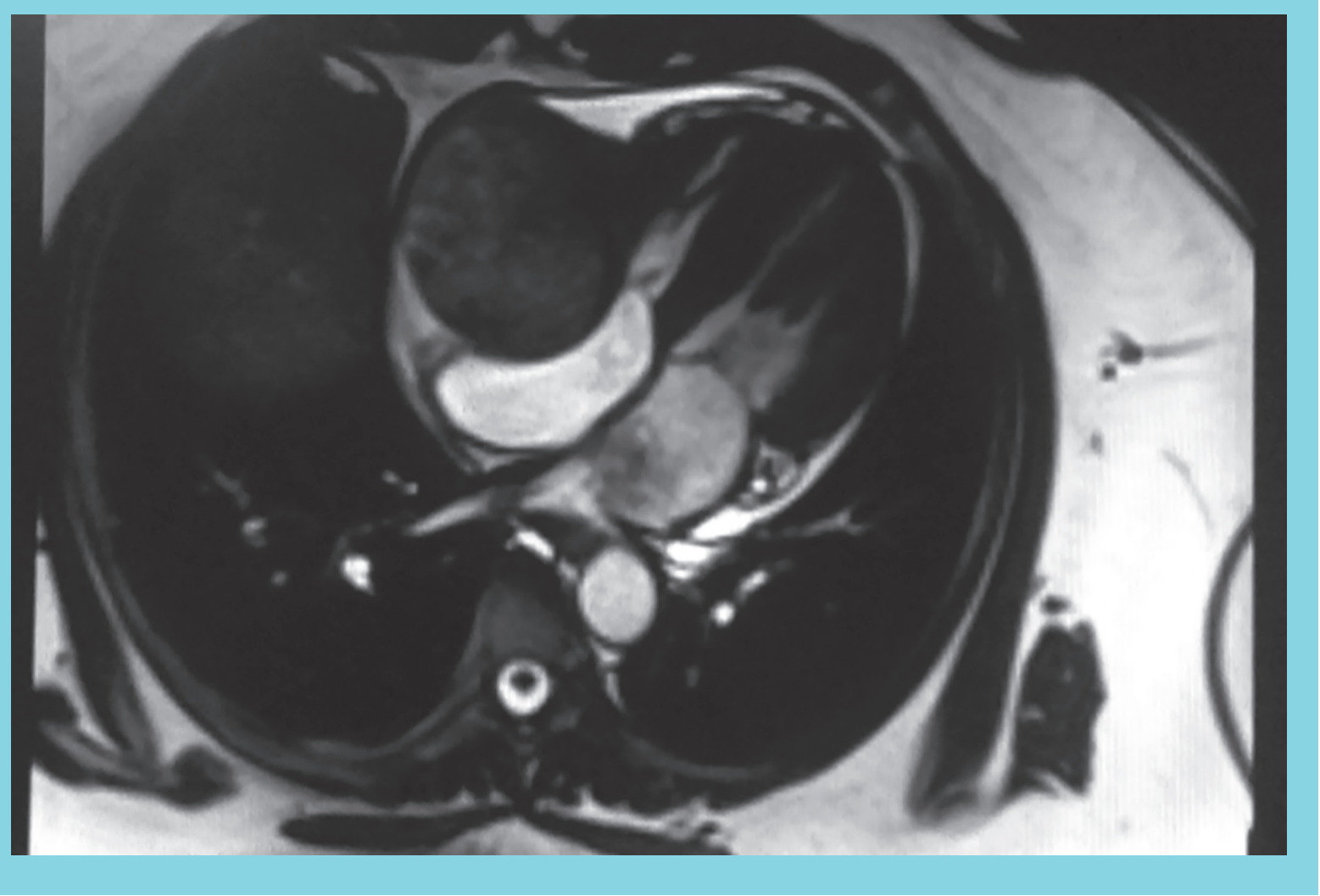


FIGURA 2: Angiografía selectiva de la arteria coronaria derecha. Se observa la extensa irrigación del tumor.

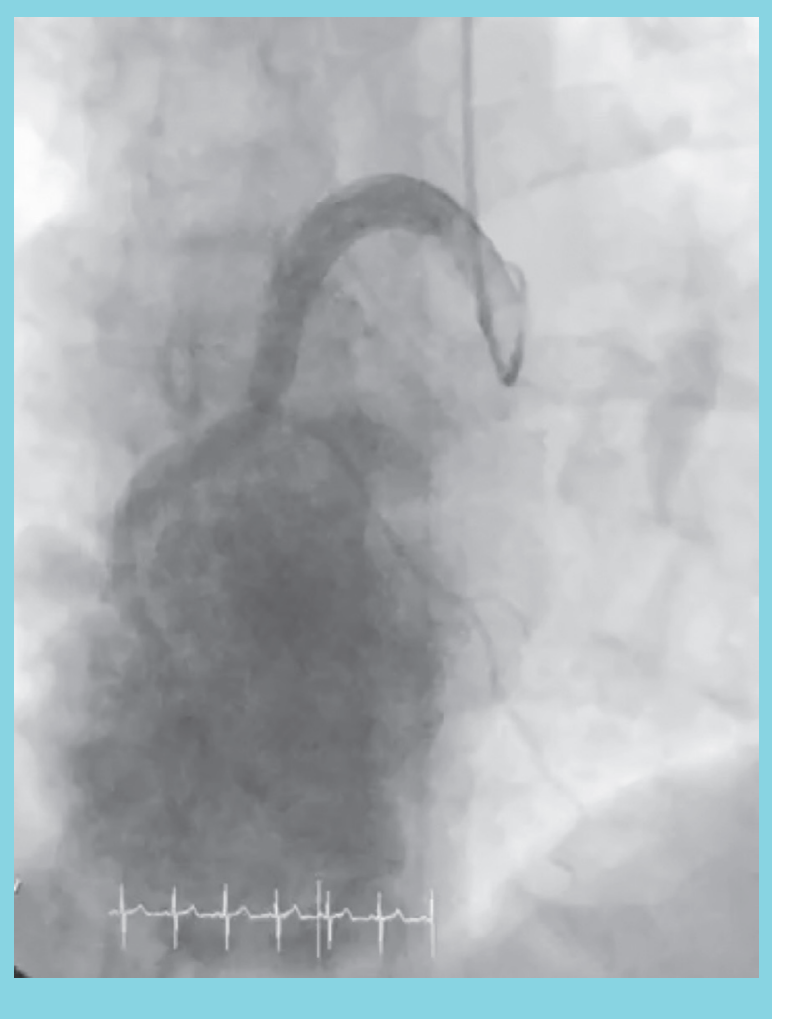

sarrollo y que se ocluye con relación al tumor (que está muy vascularizado). No se observó lecho distal de este vaso (Figura 2). La red coronaria izquierda era normal. La cirugía se realizó bajo anestesia general y por esternotomía media. En el intra operatorio el corazón se observó de tamaño, morfología y contractilidad normales. En el surco aurículo - ventricular derecho había un gran tumor sólido, lobulado, que infiltraba pared libre del ventrículo y aurícula derechos. La arteria coronaria derecha estaba comprometida en el tumor (Figura 3 ). Se resecó todo el tumor, que midió $8 \times 7 \times 3 \mathrm{~cm}$ y pesó 104gr. Externamente estaba cubierto por epicardio (Figura 4).

El defecto ventricular se cerró con puntos separados de polipropileno 4-0 apoyados en pledgets de teflón y el cierre del defecto auricular con un parche de pericardio bovino que también se fijó con sutura de polipropileno 4-0. No hubo complicaciones operatorias y la paciente fue dada de alta al sexto día post operatorio.

La anatomía patológica mostró un tumor de células epitelioides formando nidos, trabéculas y cordones, con algunas células pleomórficas (Figura 5C), que fueron positivas para marcadores neuroendocrinos (Figura 6) y parcialmente positivas para inhibina. Hubo negatividad para GFAP, calrretinina, marcadores epiteliales y mus-

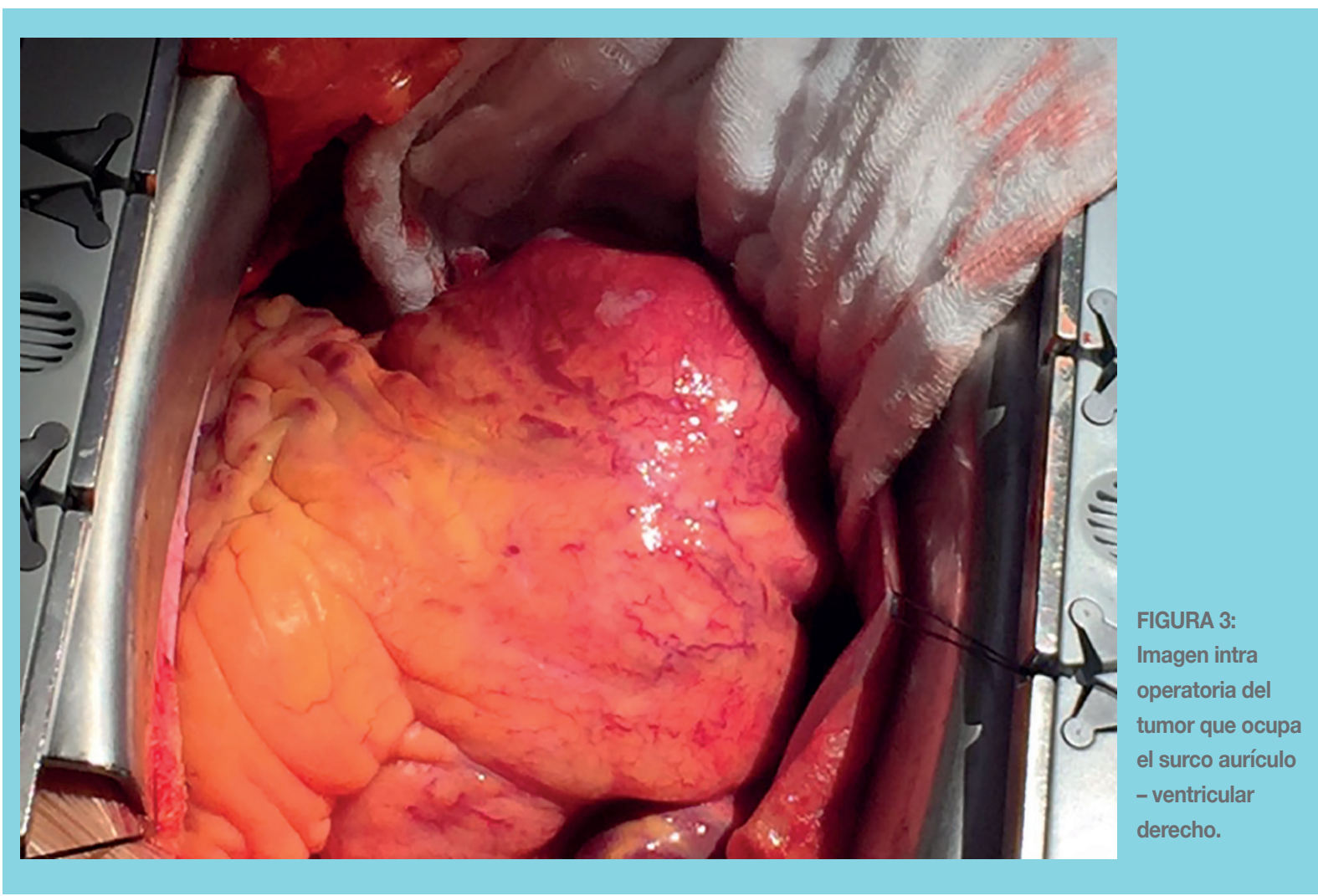




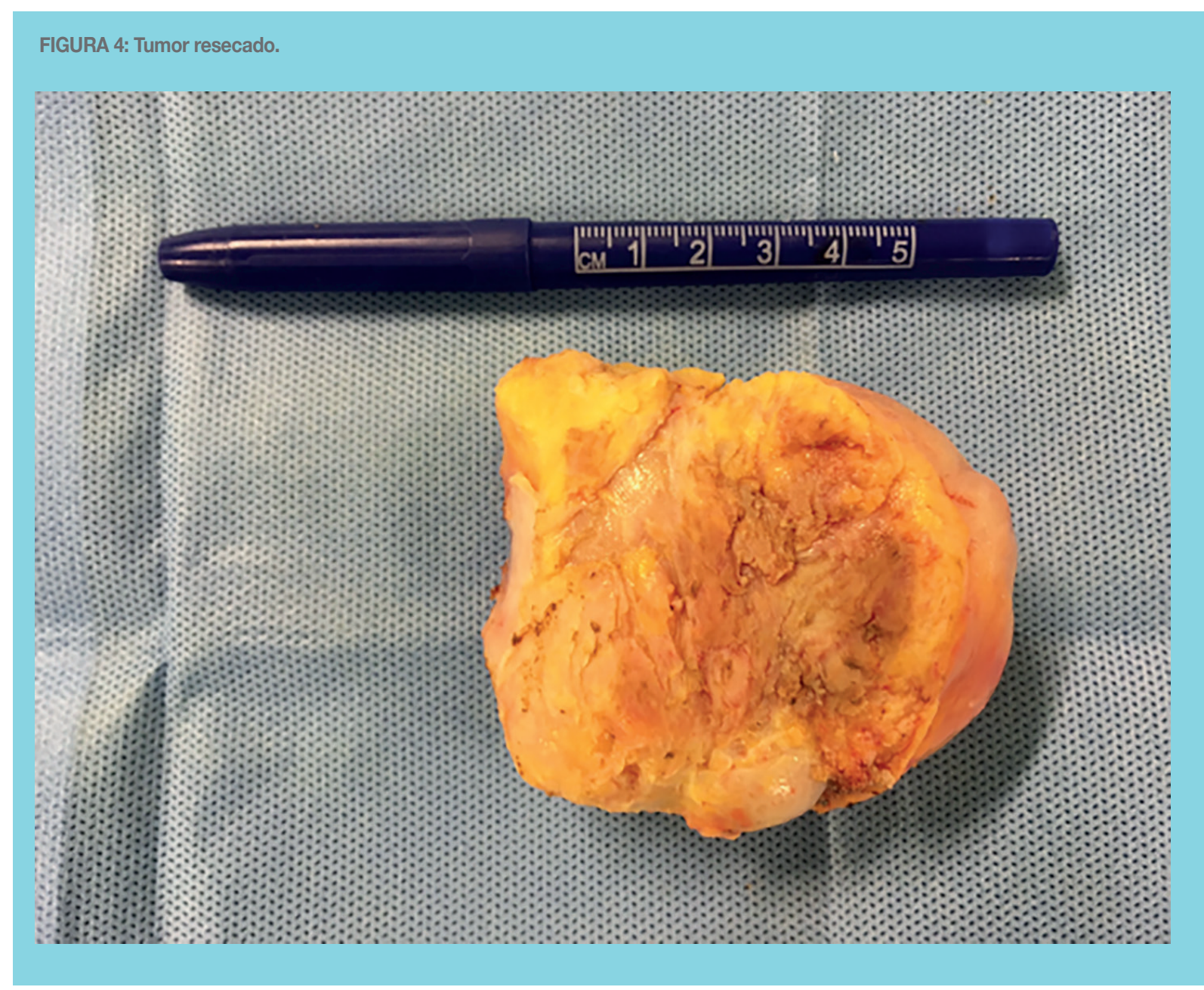

culares. Entre estas células tumorales se vieron células sustentaculares positivas para S-100. No se observaron mitosis, necrosis ni invasión linfo vascular. Estos hallazgos son compatibles con un paraganglioma. Los bordes mostraron infiltración del miocardio y pericardio (Figuras 5A, 5B).

En el seguimiento post operatorio a cuatro años la paciente permanece asintomática.

\section{Discusión:}

Los paragangliomas son tumores neuroendocrinos derivados de la cresta neural. Algunos pueden ser hormonalmente activos, productores de catecolaminas. En este caso derivan del sistema nervioso simpático y su ubicación más frecuente es la glándula adrenal, denominándose así feocromocitomas. Aquellos hormonalmente inactivos son cromafín negativos, derivan de la cadena parasimpática y se ubican con mayor frecuencia en cabeza y cuello. ${ }^{10}$
La ubicación extra adrenal de los paragangliomas es poco común. La localización torácica se observa en menos del $2 \%$ de los casos. ${ }^{11}$

La localización cardíaca es aún más infrecuente. Ésta puede ser intracardíaca o intrapericárdica, donde se localizan a nivel epicárdico. Típicamente se han descrito en el atrio izquierdo, aunque también se han observado en el septum inter atrial, en el atrio derecho, en la vena cava inferior, en la raíz de las grandes arterias y en la porción proximal de las arterias coronarias. ${ }^{9}$ En esta oportunidad, la ubicación del tumor era atípica, pues se encontraba en el surco aurículo - ventricular derecho. La presentación clínica del paraganglioma es variable, dependiendo de la ubicación y del estado funcional. En el caso de los tumores secretores de catecolaminas, el paciente cursa con hipertensión arterial, cefalea, diaforesis y palpitaciones. Cuando son funcionalmente inactivos, sólo se presentan los síntomas derivados de la compresión vascular y de la obstrucción del flujo san- 
FIGURA 5: Anatomía patológica: A) y B), miocardio y pericardio infiltrados por el tumor (H\&E 20x). C) Nidos de células tumorales (H\&E 40x).
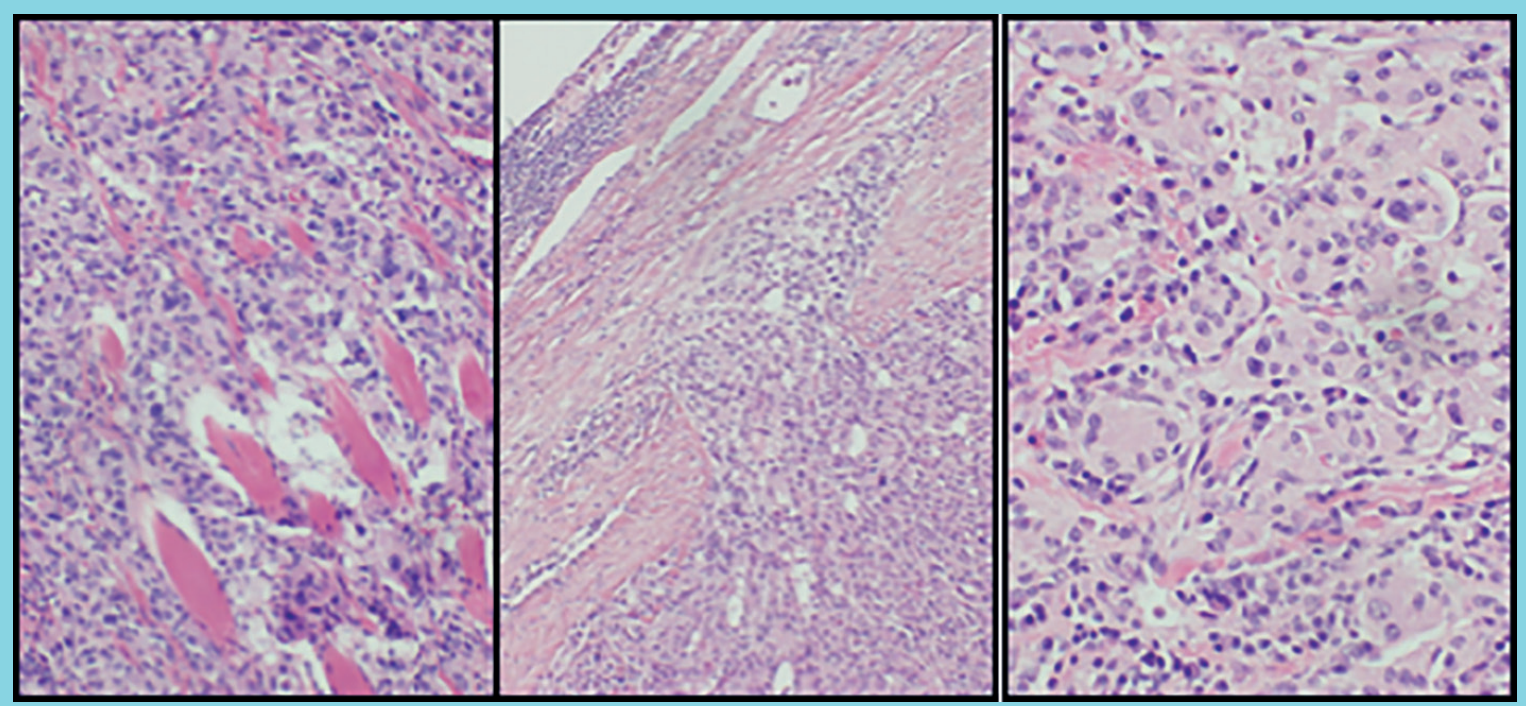

FIGURA 6: Inmunohistoquímica: A) Cromogranina; B) CD56; C) S - 100; y D) Actina, demostrando sinusoides.
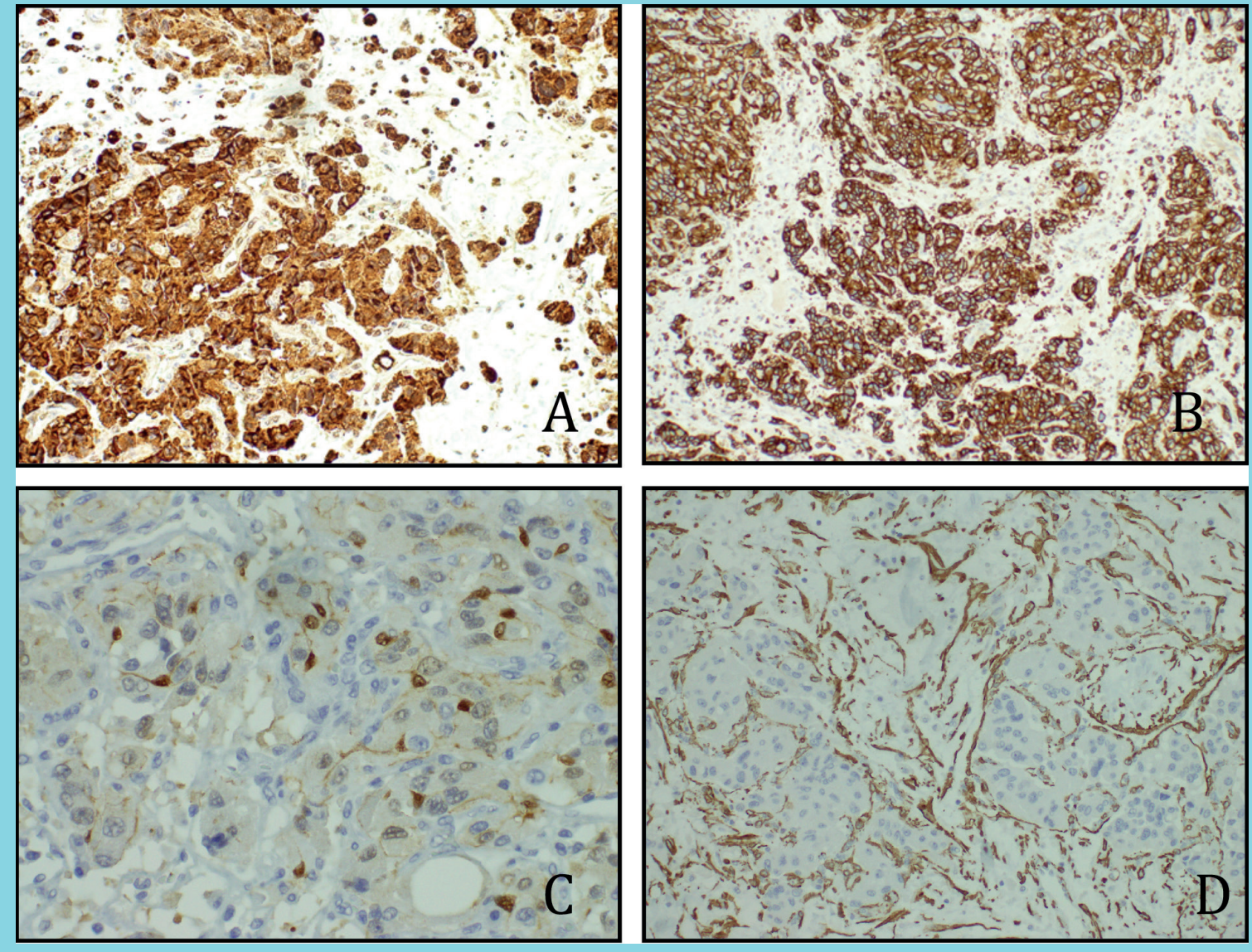
guíneo sistémico, coronario o pulmonar, cursando así con falla cardíaca o isquemia. ${ }^{12}$ En el presente caso, la paciente presentaba ambos tipos de síntomas, cursando con disnea y a su vez con palpitaciones.

Con relación al diagnóstico, la evaluación inicial consiste en una ecocardiografía, pues se trata de un examen ampliamente disponible y no invasivo que permite evaluar el miocardio, las cámaras cardíacas, la presencia del tumor y otorga información sobre la obstrucción al flujo sanguíneo. La ecocardiografía trans torácica es útil, pero se ha visto que la ecocardiografía trans esofágica sería más informativa. ${ }^{4,13}$ La resonancia magnética cardíaca entrega imágenes de alta resolución del corazón, permitiendo caracterizar la localización y apariencias radiográficas características de cada tumor. En el caso del paraganglioma, éste presenta una extrema vascularización, creando así una imagen característica. ${ }^{14}$ Una angiografía coronaria es necesaria para la planificación quirúrgica, para así poder determinar la vascularización del tumor. Típicamente, los vasos que alimentan al paraganglioma se originan en la arteria coronaria derecha, como en el caso de esta paciente, o en la arteria circunfleja izquierda. ${ }^{15}$ Adicionalmente, es importante medir catecolaminas y metanefrinas en el plasma y en orina de 24 horas, para así determinar la funcionalidad. El examen elección para determinar la localización del tumor, presencia de otros tumores y funcionalidad es el escáner con metaiodobenzylguanidina. ${ }^{12}$

Histológicamente, las células tumorales en el paraganglioma se disponen en forma de nido rodeado de estroma fibrovascular, con presencia característica de sinusoides y puede contener amiloide. El núcleo, prominente, es redondeado u oval ${ }^{16,17}$. No existen signos histológicos claros de malignidad, el único indicador es el desarrollo de metástasis ${ }^{18}$. El diagnóstico diferencial incluye otras neoplasias de distinta estirpe.
El tratamiento de elección es la resección quirúrgica. En nuestra revisión de la literatura encontramos menos de 50 casos sobre resección quirúrgica de un paraganglioma en adultos ${ }^{6}$. El abordaje puede efectuarse mediante esternotomía media, como se efectuó en este caso, o toracotomía posterolateral, según la localización del tumor. Al ser éste muy vascularizado, algunos recomiendan la embolización previa a la cirugía, para así disminuir el riesgo de complicaciones hemorrágicas.19 Además, puede ser necesario efectuar bypass cardiopulmonar e incluso paro circulatorio. También es posible que se requieran técnicas complejas de reconstrucción para así poder llevar a cabo la resección del tumor; para ello pueden emplearse parches pericárdicos bovinos o autólogos. ${ }^{8}$ En esta ocasión, se efectuó cierre del defecto auricular con parche pericárdico. En el caso de que la resección total sea extremadamente dificultosa, se ha descrito la realización de trasplante cardíaco. ${ }^{20}$

Entre el 25 y el $50 \%$ de los paragangliomas están asociados a síndromes hereditarios entre ellos la enfermedad de Von Hippel - Lindau, neurofibromatosis tipo 1, neoplasias endocrinas múltiples tipo 2A y 2B y los síndromes paraganglioma 1, 3 y 4 . Estos últimos se relacionan con la mutación de los genes que codifican para las subunidades D, A, C y B de la enzima mitocondrial succinato deshidrogenasa (SDH), trascendental para el metabolismo energético por su rol en el ciclo de Krebs. ${ }^{21}$ Así, es importante estar atento a las diferentes manifestaciones clínicas y a la historia familiar del paciente, pues mutaciones en la línea germinal fueron detectadas en el $32,1 \%$ de los pacientes con estos tumores. ${ }^{22}$ Además, cuando la SHG subunidad B se encuentra mutada, existe un mayor riesgo de malignidad; en efecto ésta se encontró alterada en el $48 \%$ de los pacientes con paraganglioma maligno. ${ }^{23}$ 


\section{Referencias}

1.- REYNEN K. Frequency of primary tumors of the heart. The American Journal of Cardiology. 1996; 77(1): 107.

2.- LAM KY, DICKENS P, CHAN AC. Tumors of the heart. A 20-year experience with a review of 12,485 consecutive autopsies. Arch Pathol Lab Med 1993; 117: 1027 -31.

3.- WANG J, WANG B, HU Y, LIU J, LIU B, LIU H et al. Clinicopathologic features and outcomes of primary cardiac tumors: a 16-year-experience with 212 patients at a Chinese medical center. Cardiovascular Pathology. 2018; 33: 45 - 54.

4.- SHAPIRO L. General Cardiology: Cardiac tumours: diagnosis and management. Heart. 2001; 85 (2): 218 -222.

5.- ARNAIZ P, TOLEDO I, BORZUTZKY A, URCELAY G, HEUSSER F, GARAY F et al . Comportamiento clínico de los tumores cardíacos desde el feto hasta el adulto: serie multicéntrica de 38 pacientes. Rev. méd. Chile; 134(9): 1135 1145 .

6.- PACHECO N, MARCOS G, GARCIPÉREZ FJ, PÉREZ C: Intrapericardial paraganglioma. Rev Esp Cardiol 2010, 63:116117.

7.- WALTHER MM, KEISER HR, LINEHAN WM: Pheochromocytoma: evaluation, diagnosis, and treatment. World J Urol 1999, 17: 35 - 39 .

8.- BURKE A, TAVORA F. The 2015 WHO Classification of Tumors of the Heart and Pericardium. Journal of Thoracic Oncology. 2016; 11(4): 441 - 452.

9.- González López M, González S, García E, Romero S, de Loma J. Surgical excision with left atrial reconstruction of a primary functioning retrocardiac paraganglioma. Journal of Cardiothoracic Surgery. 2013; 8: 22.

10.- EL-ASHRY A, CERFOLIO R, SINGH S, MCGIFFIN D. Cardiac Paraganglioma. Journal of Cardiac Surgery. 2014; 30(2):135 - 139 .

11.- ARAVOT DJ, BANNER NR, CANTOR AM, THEODOROPOULOS S, YACOUB MH. Location, locatlization and surgical treatment of cardiac pheochromocytoma. Am J Cardiol. 1992; 69: $283-5$.

12.- KHAN M, DATTA S, CHISTI M, MOVAHED M. Cardiac paraganglioma: Clinical presentation, diagnostic approach and factors affecting short and long-term outcomes. International Journal of Cardiology. 2013; 166(2): 315- 320.
13.- ENGBERDING R, DANIEL WG, ERBEL R, KASPER W, LESTUZZI C, CURTIUS JM, et al. Diagnosis of heart tumours by transoesophageal echocardiography: a multicentre study in 154 patients. European Cooperative Study Group. Eur Heart J 1993; 14:1223.

14.- FLICKINGER FW, YUH WT, BEHRENDT DM. Magnetic resonance imaging of mediastinal paraganglioma. Chest 1988; 94: 652 .

15.- WANG J, HAN J, JIANG T, LI Y. Cardiac Paragangliomas. Journal of Cardiac Surgery. 2014; 30(1): 55 - 60 .

16.- ABAD C. Tumores cardíacos (I). Generalidades. Tumores primitivos benignos. Revista Española de Cardiología. 1998; 51(1): $10-20$.

17.- LLOYD RV, OSAMURA RY, KLÖPPEL G, ROSAI J: WHO Classification of Tumours of Endocrine Organs. Fourth Edition. WHO Classification of Tumors, Volume 10. 2017. ISBN109283244931

18.- EISENHOFER G, TISCHLER AS, DE KRIJGER RR. Diagnostic tests and biomarkers for pheochromocytoma and extra-adrenal paraganglioma: from routine laboratory methods to disease stratification. Endocr Pathol 2012; $23: 4$ - 14.

19.- RAKOVICH G, FERRARO P, THERASSE E, DURANCEAU A: Preoperative embolization in the management of a mediastinal paraganglioma. Ann Thorac Surg 2001, 72: 601 -603 .

20.- JEEVANANDAM V, OZ MC, SHAPIRO B, BARR ML, MARBOE C, ROSE EA. Surgical management of cardiac pheochromocytoma. Resection versus transplantation. Ann Surg 1995; 221:415.

21.- SANCHEZ CIFUENTES A, CANDEL ARENAS M, ALBARRACÍN MARÍN-BLAZQUEZ A. Síndrome feocromocitoma-paraganglioma familiar. Medicina Clínica. 19 abril 2018. https://doi.org/10.1016/j.medcli.2018.02.019

22.- MANNELLI M, CASTELLANO M, SCHIAVI F, FILETTI S, GIACCHÈ M, MORI L et al. Clinically guided genetic screening in a large cohort of italian patients with pheochromocytomas and/or functional or nonfunctional paragangliomas. J Clin Endocrinol Metab 2009; 94:1541.

23.- CARNEY JA: CARNEY TRIAD: A syndrome featuring paraganglionic, adrenocortical, and possibly other endocrine tumors. J Clin Endocrinol Metab 2009; 94 (10): 3656 - 3662. doi: 10.1210/jc.2009-1156. Epub 2009 Sep 1. 